\title{
PCSK9 inhibition alters the lipidome of plasma and lipoprotein fractions
}

\author{
Hilvo, Mika
}

2018-02

Hilvo , M , Simolin , H , Metso , J , Ruuth , M , Öörni , K , Jauhiainen , M , Laaksonen , R \&

Baruch , A 2018 , ' PCSK9 inhibition alters the lipidome of plasma and lipoprotein fractions ' , Atherosclerosis , vol. 269 , pp. 159-165 . https://doi.org/10.1016/j.atherosclerosis.2018.01.004

http://hdl.handle.net/10138/300883

https://doi.org/10.1016/j.atherosclerosis.2018.01.004

publishedVersion

Downloaded from Helda, University of Helsinki institutional repository.

This is an electronic reprint of the original article.

This reprint may differ from the original in pagination and typographic detail.

Please cite the original version. 


\title{
PCSK9 inhibition alters the lipidome of plasma and lipoprotein fractions
}

\author{
Mika Hilvo ${ }^{a}$, Helena Simolin ${ }^{a}$, Jari Metso ${ }^{\text {b, c }}$, Maija Ruuth ${ }^{\mathrm{d}, \text { e }}$, Katariina Öörni ${ }^{\mathrm{d}}$, \\ Matti Jauhiainen b, c, Reijo Laaksonen ${ }^{\mathrm{a}, \mathrm{f}, * *, 1}$, Amos Baruch ${ }^{\mathrm{g}, *, 1}$ \\ a Zora Biosciences Oy, Biologinkuja 1, 02150, Espoo, Finland \\ ${ }^{\mathrm{b}}$ Minerva Foundation Institute for Medical Research, Biomedicum, FI-00290, Helsinki, Finland \\ ${ }^{\mathrm{c}}$ National Institute for Health and Welfare, Genomics and Biomarkers Unit, Biomedicum, FI-00290, Helsinki, Finland \\ ' Wihuri Research Institute, Haartmaninkatu 8, FI-00290, Helsinki, Finland \\ e University of Helsinki, Research Programs Unit, FI-00014, Helsinki, Finland \\ ${ }^{\mathrm{f}}$ Finnish Cardiovascular Research Center, University of Tampere and Finnish Clinical Biobank Tampere, Tampere University Hospital, Tampere, Finland \\ ${ }^{g}$ Genentech, Development Sciences, 1 DNA Way MS 46-1A, South San Francisco, CA, 94080, USA
}

\section{A R T I C L E I N F O}

\section{Article history:}

Received 12 August 2017

Received in revised form

15 November 2017

Accepted 11 January 2018

Available online 12 January 2018

\section{Keywords:}

PCSK9

Lipidomics

Lipoproteins

Atherosclerosis

Sphingolipids

Drug therapy

\begin{abstract}
A B S T R A C T
Background and aims: While inhibition of proprotein convertase subtilisin/kexin type 9 (PCSK9) is known to result in dramatic lowering of LDL-cholesterol (LDL-C), it is poorly understood how it affects other lipid species and their metabolism. The aim of this study was to characterize the alterations in the lipidome of plasma and lipoprotein particles after administration of PCSK9 inhibiting antibody to patients with established coronary heart disease.

Methods: Plasma samples were obtained from patients undergoing a randomized placebo-controlled phase II trial (EQUATOR) for the safe and effective use of RG7652, a fully human monoclonal antibody inhibiting PCSK9 function. Lipoprotein fractions were isolated by sequential density ultracentrifugation, and both plasma and major lipoprotein classes (VLDL-IDL, LDL, HDL) were subjected to mass spectrometric lipidomic profiling.

Results: PCSK9 inhibition significantly decreased plasma levels of several lipid classes, including sphingolipids (dihydroceramides, glucosylceramides, sphingomyelins, ceramides), cholesteryl esters and free cholesterol. Previously established ceramide ratios predicting cardiovascular mortality, or inflammation related eicosanoid lipids, were not altered. RG7652 treatment also affected the overall and relative distribution of lipids in lipoprotein classes. An overall decrease of total lipid species was observed in LDL and VLDL + IDL particles, while HDL-associated phospholipids increased. Following the treatment, LDL displayed reduced lipid cargo, whereas relative lipid proportions of the VLDL + IDL particles were mostly unchanged, and there were relatively more lipids carried in the HDL particles.

Conclusions: Administration of PCSK9 antibody significantly alters the lipid composition of plasma and lipoprotein particles. These changes further shed light on the link between anti-PCSK9 therapies and cardiovascular risk.
\end{abstract}

() 2018 Elsevier B.V. All rights reserved.

\footnotetext{
Abbreviations: CAD, coronary artery disease; CE, cholesteryl ester; Cer, ceramide; DAG, diacylglycerol; Gb3, globotriasoylceramide; Glc/GalCer, glucosyl/galactosylcer-

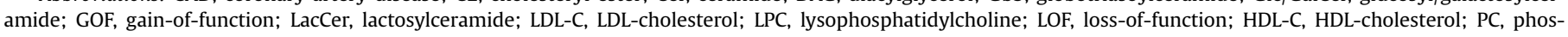
phatidylcholine; PE, phosphatidylethanolamine; PI, phosphatidylinositol; SM, sphingomyelin; TAG, triacylglycerol; TC, total cholesterol; TG, triglyceride.

* Corresponding author. Genentech Inc., 1 DNA Way, South San Francisco, CA, 94080, USA.

** Corresponding author. Zora Biosciences Oy, Biologinkuja 1, 02150, Espoo, Finland.

E-mail addresses: reijo.laaksonen@zora.fi (R. Laaksonen), baruch.amos@gene.com (A. Baruch).

1 These authors contributed equally to this work.
} 


\section{Introduction}

Atherosclerosis and coronary artery disease (CAD) are associated with increased plasma low-density lipoprotein cholesterol (LDL-C) concentration and inflammatory processes within the atherosclerotic lipid-laden lesions [1]. Statins are widely used drugs to reduce cholesterol levels and they also possess antiinflammatory properties. However, in some patients statin treatment does not result in sufficient LDL-C reduction and the use of statins is quite commonly restricted due to side effects, such as muscle pain, fatigue and weakness [2]. Moreover, statins are associated with development of diabetes [3], and therefore new drugs are being developed that would further inhibit the atherosclerotic processes with fewer side effects.

Proprotein convertase subtilisin/kexin type 9 (PCSK9) has gained interest as a new therapeutic target for coronary heart disease after it was discovered that gain-of-function (GOF) mutations in the PCSK9 gene lead to hypercholesterolemia [4] whereas loss-of-function (LOF) mutations are associated with hypocholesterolemia and reduced risk of developing coronary heart disease [5]. PCSK9 is highly expressed in liver and regulates LDL-C levels by enhancing lysosomal degradation of the hepatic lowdensity lipoprotein receptors (LDLRs) [6]. Therapeutic approaches are based on the inhibition of PCSK9 function, and the major strategies for inhibition include anti-PCSK9 antibodies, small molecule inhibitors and antisense oligonucleotide gene silencing approaches [7]. Recently, the first outcome trial with an anti-PCSK9 antibody showed reduction in non-fatal cardiovascular events [8].

While the effect of PCSK9 function on LDL-C and cholesterol metabolism has been intensively investigated, its role in metabolism of other lipid species has been underexplored, despite the evidence suggesting that inhibition of PCSK9 may cause profound changes in lipid metabolism. For instance, previous findings from our laboratory from a transgenic mouse model showed that several sphingolipid species were affected by PCSK9 deficiency [9]. Notably, in human serum several lipid species were decreased nonproportionally to LDL-C reduction $[9,10]$. This raises the possibility that PCSK9 deficiency may reduce atherosclerosis via reduction of LDL-C concentration as well as by changes in levels of other lipid classes.

To further investigate the role of PCSK9 in regulating lipid metabolism, we performed lipidomic analysis in human subjects enrolled in Phase II trial for RG7652, a fully human IgG1 monoclonal antibody that prevents the binding of PCSK9 to hepatic LDLR, thereby increasing the cell surface expression of the LDLR and enhancing LDL-C clearance. To better understand the observed changes in response to treatment with RG7652, whole plasma as well as major plasma lipoprotein classes, VLDL + IDL, LDL or HDL, were profiled in this study.

\section{Materials and methods}

\subsection{Patients and samples}

Fasting plasma samples at baseline and 29 days after treatment were obtained from 40 non-diabetic patients (Table 1) with established coronary heart disease (CHD) who were participating in a randomized, double-blind, phase II trial (EQUATOR) investigating safe and effective use of RG7652, a monoclonal antibody against PCSK9 [11]. Early time point of day 29 was chosen, as the aim was to investigate acute changes of the lipidome, after single administration of RG7652 that still reflected robust and stable LDL-lowering effect [11]. The EQUATOR trial (clinicaltrials.gov: NCT01609140) was performed in accordance with the International Conference and Harmonization guidelines and the Declaration of Helsinki, and
Table 1

Baseline characteristics of the study cohort $(\mathrm{N}=40)$.

\begin{tabular}{|c|c|c|c|}
\hline & RG7652 & Placebo & $p$-value \\
\hline No of subjects & 25 & 15 & - \\
\hline Age & $64.0(56.0-70.0)$ & $67.0(61.5-69.5)$ & 0.393 \\
\hline BMI & $29.1(24.8-31.2)$ & $29.1(26.9-31.1)$ & 0.489 \\
\hline \multicolumn{4}{|l|}{ Gender } \\
\hline Male & $15(60 \%)$ & $8(53 \%)$ & - \\
\hline Female & $10(40 \%)$ & $7(47 \%)$ & - \\
\hline \multicolumn{4}{|l|}{ Statin } \\
\hline Yes & $15(60 \%)$ & $11(73 \%)$ & - \\
\hline No & $10(40 \%)$ & $4(27 \%)$ & - \\
\hline \multicolumn{4}{|l|}{ Treatment } \\
\hline $400 \mathrm{mg} / 4 \mathrm{~W}$ & $5(20 \%)$ & $0(0 \%)$ & - \\
\hline $400 \mathrm{mg} / 8 \mathrm{~W}$ & $6(24 \%)$ & $0(0 \%)$ & - \\
\hline $800 \mathrm{mg} / 8 \mathrm{~W}$ & $11(44 \%)$ & $0(0 \%)$ & - \\
\hline $800 \mathrm{mg} / 12 \mathrm{~W}$ & $3(12 \%)$ & $0(0 \%)$ & - \\
\hline Placebo & $0(0 \%)$ & $15(100 \%)$ & - \\
\hline $\mathrm{TC}(\mathrm{mg} / \mathrm{dL})$ & $211(188-241)$ & $208(178-215)$ & 0.295 \\
\hline LDL-C (mg/dL) & $126(108-159)$ & $132(112-153)$ & 0.989 \\
\hline HDL-C (mg/dL) & $45(36-57)$ & $54(46-61)$ & 0.281 \\
\hline $\mathrm{TG}(\mathrm{mg} / \mathrm{dL})$ & $159(113-182)$ & $113(85-132)$ & 0.046 \\
\hline ApoA1 (mg/dL) & $136(122-162)$ & 145 (130-169) & 0.364 \\
\hline
\end{tabular}

Values for continuous variables represent median and interquartile range.

all patients provided written informed consent by local institutional review boards/ethics committees. The patients in this subpopulation of the EQUATOR trial were receiving four different doseregimens of RG7652 administration (Table 1) that were equally effective in lowering LDL-C [11], and for the statistical analyses all samples from treated groups were pooled into a single set of patients. Altogether 25 patients were receiving RG7652 and 15 placebo (Table 1). The criteria for patient selection was as follows: no diabetes, chronic kidney disease, or familiar hypercholesterolemia and all were Caucasians. RG7652 and placebo groups for the study were well matched for cholesterol levels, however, a slight imbalance was evident in triglyceride (TG) levels (Table 1 ).

\subsection{Lipoprotein isolation from human plasma}

Major lipoprotein classes were isolated from plasma samples by sequential ultracentrifugation using table-top ultracentrifuge (Beckmann Optima TL, USA) and $\mathrm{KBr}$ for density adjustment [12]. Serum sample $(0.18-0.3 \mathrm{~mL})$ was first adjusted to the density $(\mathrm{d})$ of $1.019 \mathrm{~g} / \mathrm{mL}$ and the centrifuge tube filled with a $\mathrm{d}=1.019 \mathrm{~g} / \mathrm{mL} \mathrm{KBr}$ solution to the total volume of $3 \mathrm{~mL}$. The samples were centrifuged at $5{ }^{\circ} \mathrm{C}$ for $2 \mathrm{~h}$ at the speed $100,000 \mathrm{rpm}$ (corresponding to relative centrifugal force of $500,000 \times g$ ). After centrifugation, very low and intermediate density lipoproteins (VLDL + IDL) were recovered in the top $1 \mathrm{~mL}$ fraction and the infranatant fraction was adjusted to the density of $1.063 \mathrm{~g} / \mathrm{mL}$ using solid $\mathrm{KBr}$, filled again to final volume of $3 \mathrm{~mL}$ with $\mathrm{d}=1.063 \mathrm{~g} / \mathrm{mL} \mathrm{KBr}$ solution and centrifuged $\left(5{ }^{\circ} \mathrm{C}, 3 \mathrm{~h}, 100,000 \mathrm{rpm}\right)$. The top $1 \mathrm{~mL}$ fraction contained low density lipoprotein (LDL) particles. To get the total HDL fraction the infranatant fraction was adjusted with $\mathrm{KBr}$ to the density of $1.21 \mathrm{~g} /$ $\mathrm{mL}$, the vials filled with $\mathrm{KBr}$ density $(1.21 \mathrm{~g} / \mathrm{mL})$ solution and then centrifuged $\left(5^{\circ} \mathrm{C}, 18 \mathrm{~h}, 100,000 \mathrm{rpm}\right)$. Total HDL was obtained in top $1 \mathrm{~mL}$ fraction. The isolated fractions were stored at $-80^{\circ} \mathrm{C}$ before analysis.

\subsection{Lipidomic analysis of plasma and lipofractions}

Lipids from total plasma and VLDL + IDL, LDL and HDL particles were extracted using a modified Folch lipid extraction [13,14] performed on a Hamilton Microlab Star robot. Samples were spiked with known amounts of non-endogenous synthetic internal standards. After lipid extraction, lipids were resolubilized in 
chloroform:methanol $(1: 2, \mathrm{v} / \mathrm{v})$ and synthetic external standards were added to the lipid extracts. The extracts were stored at $-20^{\circ} \mathrm{C}$ prior to mass spectrometry (MS) analysis. For quantification of free cholesterol (FC) from plasma, an aliquot of each lipid extract was treated with acetyl chloride to derivatize FC to modified cholesteryl ester species.

In Shotgun Lipidomics, lipid extracts were analyzed using a hybrid triple quadrupole/linear ion trap mass spectrometer (QTRAP 5500 ) equipped with a robotic nanoflow ion source (NanoMate HD) according to Ståhlman and colleagues [15]. Molecular lipids were analyzed in both positive and negative modes using Multiple Precursor Ion Scanning (MPIS) based methods [16,17]. In plasma samples, phospholipid species were analyzed with shotgun lipidomics, while in isolated lipoprotein fractions, phospholipids were analyzed with an ultra-high pressure liquid chromatography (UHPLC) system (Shimadzu Nexera 2 UHPLC or Eksigent XL-110 system) using multiple reaction monitoring (MRM) -based method in negative ion mode. Triacylglycerols (TAG) were analyzed using Precursor Ion Scanning (PIS) and Neutral Loss scanning (NL) based methods. The molecular lipid species were identified and quantified in semi-absolute or absolute amounts [15]. Ceramides were analyzed on a hybrid triple quadrupole/linear ion trap mass spectrometer (5500 QTRAP) equipped with an ultra-high pressure liquid chromatography (UHPLC) system (CTC HTC PAL autosampler and Rheos Allegro pump) using multiple reaction monitoring (MRM) - based method in negative ion mode. Lipids were normalized to their respective internal standard and the sample amount. The concentrations of molecular lipids were presented as $\mu \mathrm{M}$. In lipoprotein fractions, lipids were also normalized with total protein content of the lipoprotein particles (Micro BCA Protein Assay Kit, Thermo Scientific).

\subsection{Statistical analysis}

Statistical analyses were performed using SAS version 9.4 and $\mathrm{R}$ version 3.3.0. Due to smaller number of subjects than in the original EQUATOR trial [11] and a slight imbalance of statin use in the RG7652 and placebo groups, instead of the group-wise comparisons the statistical analyses were performed with paired $t$-test as time-wise comparisons, using log-transformed concentrations. Data were visualized using Tableau Desktop 8.2.1 and the percentage differences were calculated with pairwise comparisons: presented differences are relative differences between group mean values.

\section{Results}

\subsection{PCSK9 inhibition impacts plasma sphingolipids and cholesterol esters}

Lipidomic analysis was performed from patients receiving antiPCSK9 therapy (RG7652) or placebo for 29 days (Table 1). Congruent with previous report [11], 29 days after initiation of treatment with RG7652, a marked decrease was observed in plasma levels of LDL-C, non-HDL-cholesterol (Non-HDL-C) and apolipoprotein B-100 (apoB) while HDL-C was slightly increased (Fig. 1A). No significant changes were detected in the placebo group. Overall, administration of RG7652 resulted in decrease of most lipid classes in plasma (Fig. 1B) and the results were consistent in subjects with and without statin therapy (Supplementary Fig. 1). The most affected lipid classes were sphingolipids including dihydroceramides (Cer d18:0), globotriasoylceramides (Gb3), glucosyl/galactosylceramides (Glc/GalCer), sphingomyelins (SM), ceramides (Cer d18:1) and lactosylceramides (LacCer) (Fig. 1B, Supplementary Fig. 2). A marked reduction was also observed in cholesteryl esters
(CE) and free cholesterol (FC). Phospholipids as well as tri- and diacylglycerols (TAG, DAG) were also reduced, but to a lesser extent as compared with sphingolipids or CEs.

Several ceramide classes appeared to have a common trend where, following PCSK9 inhibition, lipids with longer fatty acyl side chains were reduced to a greater extent as compared to their shorter fatty acyl side chain counterparts (Supplementary Fig. 2). Consistent with this observation, ceramides containing very long saturated fatty acid (22:0, 24:0 and 26:0) had a more substantial reduction upon inhibition of PCSK9 when compared with the other ceramide species (Fig. 1C). This same trend was not apparent for other lipid classes such as sphingomyelins or cholesteryl esters (Supplementary Fig. 2). We have recently reported that specific ceramide ratios [Cer(d18:1/16:0)/Cer(d18:1:/24:0), Cer(d18:1/ 18:0)/Cer(d18:1:/24:0) and $\operatorname{Cer}(\mathrm{d} 18: 1 / 24: 1) / \operatorname{Cer}(\mathrm{d} 18: 1: / 24: 0)]$ have strong predictive value for fatal cardiovascular events [18], however, these ratios were not significantly altered due to the PCSK9 inhibition (Supplementary Fig. 2). In addition, eicosanoids did not show major changes after RG7652 treatment.

\subsection{Effect of PCSK9 inhibition on the lipidome of lipoproteins}

The observed variable decrease of different sphingolipid and cholesteryl ester species in plasma suggest that PCSK9 inhibition does not solely decrease LDL particle number, but may also affect the lipid composition of the lipoproteins. To test that, we isolated VLDL + IDL, LDL and HDL lipoprotein classes from plasma samples followed by lipidomic profiling. First, we investigated how circulating lipid load is shifted between lipoprotein particles due to PCSK9 inhibition. As the PCSK9 inhibition appeared to have a different effect on various ceramides in plasma, we analyzed their relative distribution in the isolated lipoprotein classes at baseline (Fig. 1D). Non-saturated 24:1-containing ceramides were shown to be less abundant than 24:0- and 26:0-containing ceramides in LDL particles, explaining why PCSK9 inhibition decreases less 24:1 containing ceramides than those with long saturated fatty acids in plasma. When investigating the total lipid shift between lipoprotein particles, for all lipid classes the conclusion was the same; after RG7652 treatment LDL particles carried less lipids, while lipid proportions in VLDL + IDL were rather unchanged, and there was relatively higher amount of lipids carried in the HDL particles (Fig. 2A).

Next, we normalized the lipidomic data to the total protein content of each lipoprotein fraction to account for changes in particle numbers. An overall decrease was observed on lipid class level in LDL and VLDL + IDL fractions after PCSK9 inhibition (Fig. 2B), while no significant changes were observed in the placebo group (Supplementary Fig. 3). The most pronounced reduction was recorded for CEs and sphingolipids in both apoB-containing lipoprotein fractions; in LDL fraction, phosphatidylcholines (PC) also showed a strong decrease. In HDL particles, ceramides and dihydroceramides were predominantly decreased, while phospholipids (PCs and phosphatidylethanolamines (PEs)) were increased after PCSK9 inhibition.

As suggested by the total lipid class results presented above, levels of practically all individual lipid species were reduced in the LDL and VLDL + IDL particles due to PSCK9 inhibition (Supplementary Fig. 4). However, more variation was recorded among the lipid species in HDL particles. For instance, some SM species [SM (d18:1/16:0), SM (d18:1/16:1) and SM (d18:1/18:1)] were increased in HDL while these SM species were strongly reduced in VLDL + IDL and LDL fractions. Furthermore, linoleic acid (18:2) and arachidonic acid (20:4) containing phosphatidylcholines (PCs) and phosphatidylethanolamines (PEs) were increased in the HDL particles after PCSK9 inhibition. Finally, the aforementioned 

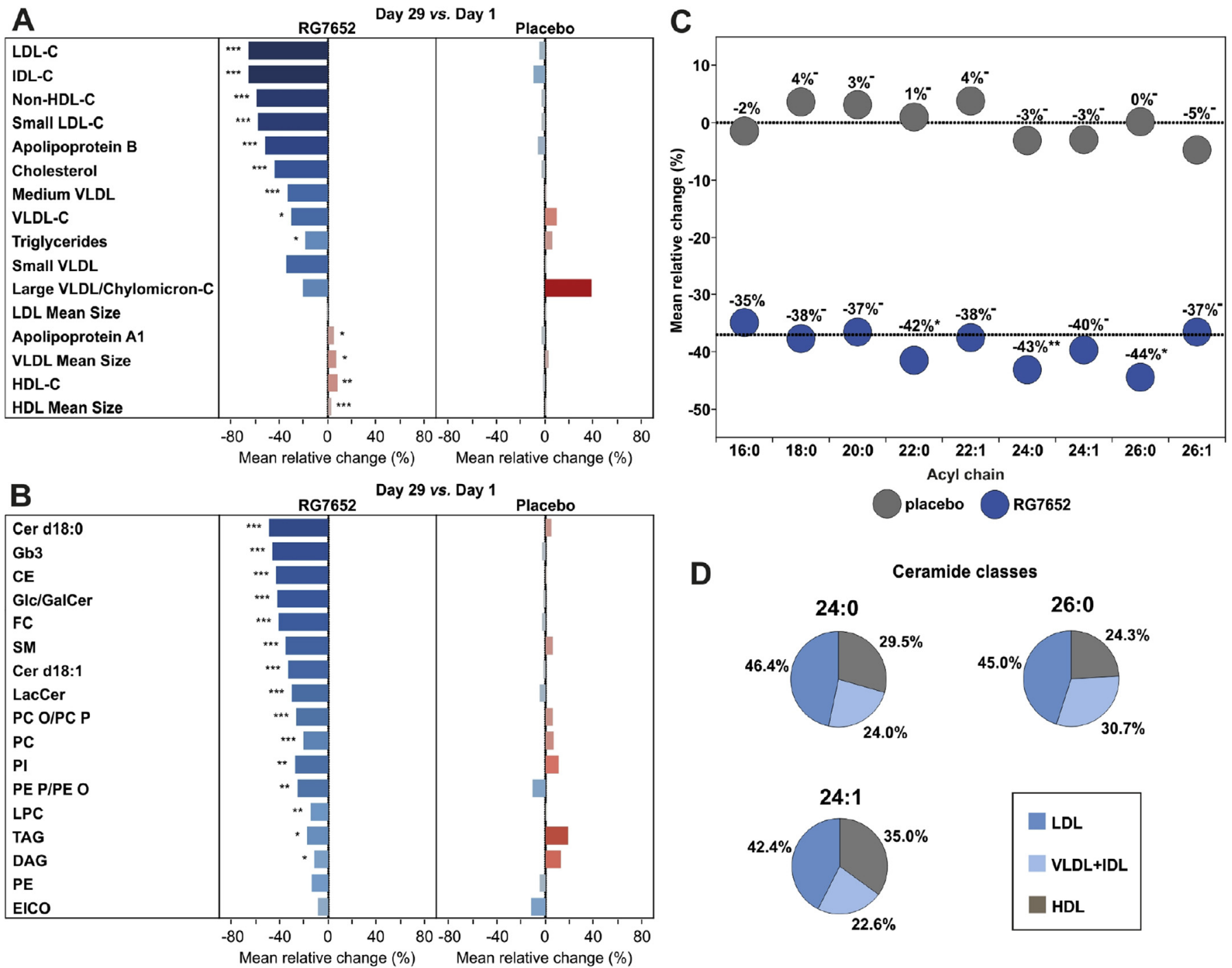

eramide classes
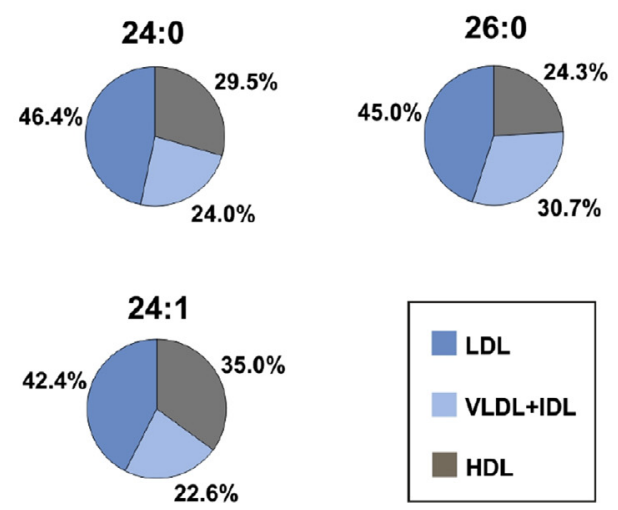

Fig. 1. Changes of clinical variables and lipids in RG7652 and placebo groups.

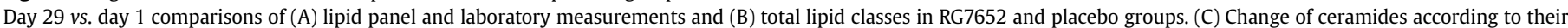

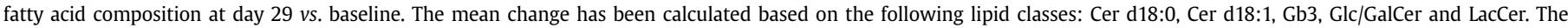

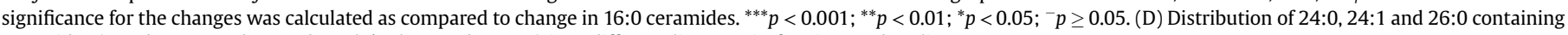
ceramides (Cer d18:0, Cer d18:1, Gb3, Glc/GalCer and LacCer) into different lipoprotein fractions at baseline.

ceramide ratios related to cardiovascular death were elevated in the HDL particles.

To further investigate the relative changes in the lipid composition of different lipoprotein particles we calculated the relative percentage of all lipid classes by dividing their concentration by the total lipid concentration in the distinct lipoprotein fraction. In general, CE concentration seemed to decrease in all lipoprotein fractions (Table 2). This effect was particularly obvious in the VLDL + IDL fraction, where a significant relative decrease of 30\% was observed. Another common trend in all lipoprotein fractions was significant relative increase in different phospholipid classes (PC, PE, PI). Finally, the relative amount of triacylglycerols was significantly increased in LDL and VLDL + IDL fractions.

\section{Discussion}

This study is the first one to characterize the lipidome of plasma and isolated lipoprotein fractions following treatment with a PCSK9 inhibitor. PCSK9 inhibition resulted in significant decreases in plasma levels of several lipid classes, especially sphingolipids and cholesteryl esters, and also affected the overall and relative distribution of hundreds of different lipid species within and between lipoprotein particles. The data reported here is consistent with previously reported lipidomic data in plasma from PCSK9-deficient mice and humans harboring a LOF mutation (R46L) in the PCSK9 gene [9]. Mice on a regular chow diet showed a strong reduction of sphingolipids and cholesteryl esters, in line with the present human study. In humans with a PCSK9 LOF mutation, several lipid classes including sphingolipids and cholesteryl esters showed 15-25\% lower levels as compared to subjects with wild type PCSK9, while the difference for LDL-C was more modest (10\%) [9]. Thus, the remaining important question is whether reduction of biologically active lipids, LDL-C, or both is more relevant and clinically sufficient for the outcome of the patients.

Previous studies have shown that specific lipids, impacted also by PCSK9 inhibition, can alter the progression of atherosclerosis and their plasma levels can introduce further added predictive value for the outcome of coronary artery disease [18-20]. Ceramide 

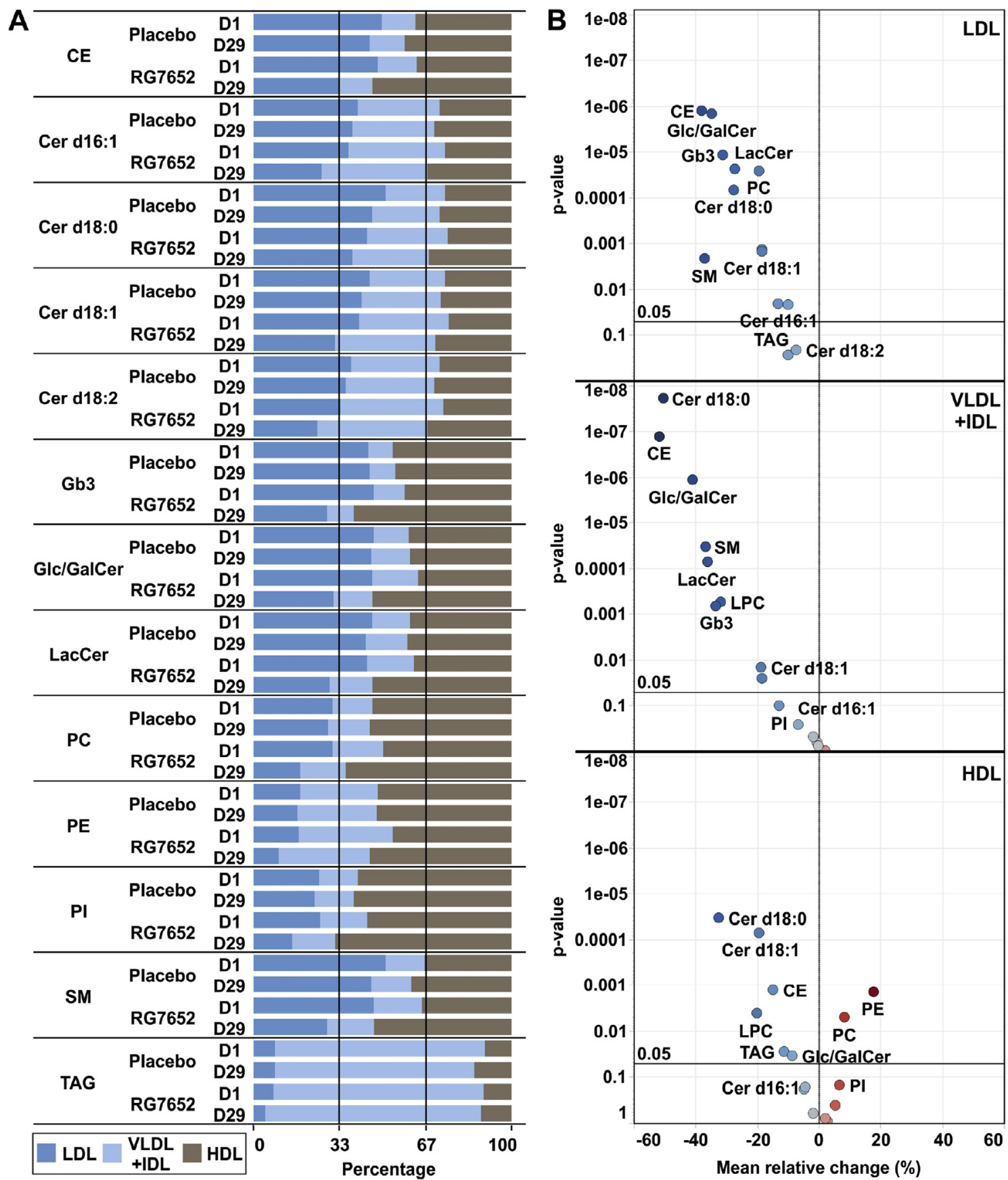

Fig. 2. Lipid class distributions and changes in lipoprotein classes.

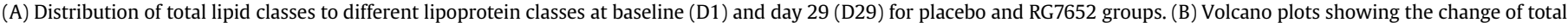
lipid classes at day $29 \mathrm{vs}$. baseline for lipidomic data normalized with total protein content of each lipoprotein class.

ratios, including Cer(d18:1/24:1)/Cer(d18:1/24:0), predict especially fatal cardiovascular events in patients with stable coronary artery disease and acute coronary syndrome [18]. Interestingly, the degree of reduction of plasma ceramides in response to PCSK9 inhibition was dependent on ceramide fatty acyl chain length, or even the number of double bonds. The reason for this phenomenon was difference in the distribution of the ceramides in the lipoprotein fractions. As an example, 24:1 fatty acid containing ceramides were relatively less abundant in LDL than those incorporating 24:0 fatty acid, leading to nonsignificant change of this cardiovascular death associated plasma ceramide ratio after dramatic LDL-C lowering. Interestingly, recent data from the FOURIER outcome trial show that while prolonged PCSK9 inhibition significantly reduces nonfatal CV events, there was no improvement in the risk reduction of fatal cardiovascular events [8]. However, our previous results from humans and mice with LOF in the PCSK9 gene showed greater decrease for $\operatorname{Cer}(\mathrm{d} 18: 1 / 16: 0)$ and $\operatorname{Cer}(\mathrm{d} 18: 1 / 18: 0)$ than for Cer(d18:1/24:0) [9], suggesting that modulation of PCSK9 activity by antibodies or genetic background may affect the cardiovascular death associated ceramide ratios differently. Although such link between lipidomic changes and cardiovascular mortality requires further validation, it suggests that lipid biomarkers, beyond LDL-C, may hold important clues on the clinical progression of cardiovascular disease. 
Table 2

Relative (\%) lipid class composition of lipoprotein fractions at baseline (D1) and day 29 (D29).

\begin{tabular}{|c|c|c|c|c|c|c|c|c|c|c|c|c|c|c|c|}
\hline \multirow[t]{3}{*}{ Lipid class } & \multicolumn{5}{|l|}{ LDL } & \multicolumn{5}{|c|}{ VLDL + IDL } & \multicolumn{5}{|l|}{$\mathrm{HDL}$} \\
\hline & \multicolumn{2}{|l|}{ RG7652 } & \multirow[t]{2}{*}{ Change (\%) } & \multicolumn{2}{|l|}{ Placebo } & \multicolumn{2}{|c|}{ RG7652 } & \multirow[t]{2}{*}{ Change $(\%)^{\mathrm{a}}$} & \multicolumn{2}{|c|}{ Placebo } & \multicolumn{2}{|l|}{ RG7652 } & \multirow[t]{2}{*}{ Change (\%) } & \multicolumn{2}{|l|}{ Placebo } \\
\hline & D1 & D29 & & D1 & D29 & D1 & D29 & & D1 & D29 & D1 & D29 & & D1 & D29 \\
\hline $\mathrm{CE}$ & 82.1 & 77.7 & -5.4 & 81.6 & 81.7 & 45.9 & 32.1 & -30.0 & 45.8 & 46.5 & 70.2 & 65.5 & -6.6 & 70.5 & 70.6 \\
\hline PC & 12.3 & 16.3 & 32.4 & 12.5 & 12.5 & 14.3 & 16.2 & 13.0 & 14.3 & 14.3 & 23.1 & 30.2 & 30.7 & 28.1 & 22.8 \\
\hline TAG & 1.5 & 2.3 & 53.2 & 1.4 & 1.5 & 35.9 & 47.7 & 33.0 & 35.8 & 35.2 & 3.1 & 3.0 & n.s. & 2.7 & 2.7 \\
\hline SM & 3.4 & 3.2 & n.s. & 3.8 & 3.7 & 2.3 & 2.1 & -10.3 & 2.4 & 2.4 & 2.9 & 3.1 & n.s. & 2.9 & 3.0 \\
\hline Cer d16:1 & 0.0083 & 0.0118 & 42.7 & 0.0082 & 0.0076 & 0.016 & 0.020 & 26.4 & 0.014 & 0.014 & 0.0066 & 0.0072 & n.s. & 0.0065 & 0.0056 \\
\hline Cer d18:0 & 0.0017 & 0.0019 & n.s. & 0.0018 & 0.0016 & 0.002 & 0.002 & -26.7 & 0.002 & 0.002 & 0.0011 & 0.0009 & -19.2 & 0.0010 & 0.0009 \\
\hline Cer d18:1 & 0.124 & 0.164 & 32.1 & 0.140 & 0.127 & 0.194 & 0.213 & 9.6 & 0.196 & 0.201 & 0.083 & 0.078 & n.s. & 0.092 & 0.078 \\
\hline Cer d18:2 & 0.015 & 0.023 & 53.3 & 0.017 & 0.015 & 0.033 & 0.043 & 30.0 & 0.035 & 0.033 & 0.013 & 0.015 & n.s. & 0.015 & 0.012 \\
\hline DAG & - & - & - & - & - & 0.410 & 0.551 & n.s & 0.425 & 0.416 & 0.034 & 0.037 & n.s. & 0.029 & 0.030 \\
\hline Gb3 & 0.028 & 0.031 & n.s. & 0.030 & 0.031 & 0.013 & 0.012 & n.s & 0.013 & 0.013 & 0.028 & 0.034 & 22.2 & 0.038 & 0.030 \\
\hline Glc/GalCer & 0.19 & 0.20 & n.s. & 0.18 & 0.19 & 0.13 & 0.10 & -19.4 & 0.11 & 0.12 & 0.17 & 0.18 & n.s. & 0.18 & 0.15 \\
\hline LacCer & 0.12 & 0.14 & 14.6 & 0.14 & 0.14 & 0.09 & 0.08 & -9.8 & 0.10 & 0.09 & 0.12 & 0.13 & n.s. & 0.15 & 0.12 \\
\hline LPC & - & - & - & - & - & 0.30 & 0.29 & n.s & 0.29 & 0.31 & 0.13 & 0.11 & n.s. & 0.15 & 0.14 \\
\hline $\mathrm{PE}$ & 0.09 & 0.14 & 53.7 & 0.11 & 0.10 & 0.33 & 0.47 & 41.2 & 0.40 & 0.39 & 0.26 & 0.37 & 30.7 & 0.37 & 0.30 \\
\hline PI & 0.07 & 0.10 & 37.3 & 0.07 & 0.08 & 0.09 & 0.11 & 19.4 & 0.10 & 0.10 & 0.19 & 0.24 & 27.6 & 0.23 & 0.19 \\
\hline
\end{tabular}

Placebo group did not show significant changes in any of the comparisons.

a n.s., not significant $(p \geq 0.05)$.

The results of this study not only highlight the change in different lipid levels in LDL particles after PCSK9 inhibition, but also that the relative lipid composition of the LDL, VLDL and HDL particles is altered. When looking at the relative lipid distribution within the particles, a common phenomenon in all major lipoprotein classes was the systematic reduction of cholesteryl esters which resulted in an increase in the other lipid classes. However, when investigating the lipids normalized to total protein content of the lipoproteins, it appeared that VLDL and LDL fractions had significantly decreased levels of most of the lipid classes, suggesting a decrease in their sizes, while in HDL fractions, phospholipids were increased. The substantial decrease of lipids versus protein ratio in VLDL and LDL could imply that PCSK9 inhibition affects the lipid content of the particles, particle size or the distribution of specific lipoprotein classes. Although not observed in this substudy, analysis of the entire study population show a significant reduction in LDL particle size following treatment with anti-PCSK9 [11]. In a separate study an increase in HDL as well as VLDL particle size was detected following PCSK9 inhibition [21]. It still remains to be determined how these changes impact the functionality of the lipoprotein particles. A potential limitation of our study is the lack of proteomic data that could provide further information on the changes in lipoprotein classes following marked reduction of LDLC.

Previous results have reported that PCSK9 inhibition, while markedly reducing LDL-C, does not affect inflammatory markers, such as CRP and pro-inflammatory cytokines [11,22]. Our current finding demonstrating that the inflammation-related eicosanoids [23] did not show major alterations may provide a mechanistic explanation for this clinical observation. Nevertheless, in HDL particles phospholipid species containing the inflammation related arachidonic acid (20:4), and its precursor linoleic acid (18:2), were increased more than the other phospholipid species. An increase of these fatty acids in HDL particles may indicate that the arachidonic acid in HDL particles is neutralized via hepatic HDL particle uptake and degradation, thereby suggesting possible anti-inflammatory effect on the cardiovascular system. However, additional studies are needed to understand the significance of our observation.

In conclusion, our study revealed that antibody-mediated PCSK9 inhibition does not only affect LDL cholesterol metabolism, but also alters the global lipidome both at the level of plasma and circulating lipoprotein particles. Our results are also consistent with lipidomic alterations that have been described due to statin treatment, as e.g. rosuvastatin has been shown to decrease sphingolipids more than phospholipids, similar to the results of the present study [24]. This suggests that although statins and anti-PCSK9 inhibitors work through different mechanism of actions, the major driver for the observed treatment-related effect on the lipidome is their overall LDL-lowering effect. Taken together the present and previous results, it has become apparent that LDL-cholesterol lowering therapies affect profoundly the plasma and lipoprotein lipidome. It would be important to understand the relative importance of all the other alterations in the lipidome, as compared to the reduction of cholesterol content, in the prevention of cardiovascular events. This can be achieved only by systematic analysis on how the full lipidome of plasma and lipoprotein particles both predict cardiovascular risk and how modifications of the lipidome by PCSK9, statin or other lipid modifying therapies affect the outcome of the patients.

\section{Conflict of interest}

Zora Biosciences holds patents for the diagnostic use of ceramides, and R.L. is a shareholder of Zora Biosciences. M.H., H.S. and R.L. are employees of Zora Biosciences. The other authors report no conflicts.

\section{Financial support}

M.R. and K.Ö. were supported by Finnish Foundation for Cardiovascular Research and K.Ö. by Academy of Finland (grant number 265940). M.J. and J.M. were supported by Jane and Aatos Erkko Foundation (\#01062016).

\section{Author contributions}

Wrote manuscript: M.H., A.B., R.L., K.Ö., M.J.; designed research: A.B., R.L., M.H.; performed research: H.S., J.M., M.R.; analyzed data: M.H., A.B., R.L., K.Ö., M.J.

\section{Appendix A. Supplementary data}

Supplementary data related to this article can be found at https://doi.org/10.1016/j.atherosclerosis.2018.01.004. 


\section{References}

[1] G.K. Hansson, Inflammation, atherosclerosis, and coronary artery disease N. Engl. J. Med. 352 (2005) 1685-1695, https://doi.org/10.1056/ NEJMra043430.

[2] B.A. Golomb, M.A. Evans, Statin adverse effects: a review of the literature and evidence for a mitochondrial mechanism, Am. J. Cardiovasc. Drugs 8 (2008) 373-418, https://doi.org/10.2165/0129784-200808060-00004.

[3] P.D. Thompson, G. Panza, A. Zaleski, B. Taylor, Statin-associated side effects, J. Am. Coll. Cardiol. 67 (2016) 2395-2410, https://doi.org/10.1016 j.jacc.2016.02.071.

[4] M. Abifadel, M. Varret, J.-P. Rabès, D. Allard, K. Ouguerram, M. Devillers, C. Cruaud, S. Benjannet, L. Wickham, D. Erlich, A. Derré, L. Villéger, M. Farnier, I. Beucler, E. Bruckert, J. Chambaz, B. Chanu, J.-M. Lecerf, G. Luc, P. Moulin, J. Weissenbach, A. Prat, M. Krempf, C. Junien, N.G. Seidah, C. Boileau, Mutations in PCSK9 cause autosomal dominant hypercholesterolemia, Nat. Genet. 34 (2003) 154-156, https://doi.org/10.1038/ng1161.

[5] J.C. Cohen, E. Boerwinkle, T.H. Mosley, H.H. Hobbs, Sequence variations in PCSK9, low LDL, and protection against coronary heart disease, N. Engl. J. Med 354 (2006) 1264-1272, https://doi.org/10.1056/NEJMoa054013.

[6] A.S. Peterson, L.G. Fong, S.G. Young, PCSK9 function and physiology, J. Lipid Res. 49 (2008) 1595-1599, https://doi.org/10.1194/jlr.E800008-JLR200.

[7] G.D. Norata, G. Tibolla, A.L. Catapano, Targeting PCSK9 for hypercholesterolemia, Annu. Rev. Pharmacol. Toxicol. 54 (2014) 273-293, https://doi.org 10.1146/annurev-pharmtox-011613-140025.

[8] M.S. Sabatine, R.P. Giugliano, A.C. Keech, N. Honarpour, S.D. Wiviott, S.A. Murphy, J.F. Kuder, H. Wang, T. Liu, S.M. Wasserman, P.S. Sever, T.R. Pedersen, Evolocumab and clinical outcomes in patients with cardiovascular disease, N. Engl. J. Med. (2017), https://doi.org/10.1056/NEJMoa1615664. NEJMoa1615664.

[9] M.T. Jänis, K. Tarasov, H.X. Ta, M. Suoniemi, K. Ekroos, R. Hurme, T. Lehtimäki, H. Päivä, M.E. Kleber, W. März, A. Prat, N.G. Seidah, R. Laaksonen, Beyond LDLC lowering: distinct molecular sphingolipids are good indicators of proprotein convertase subtilisin/kexin type 9 (PCSK9) deficiency, Atherosclerosis 228 (2013) 380-385, https://doi.org/10.1016/j.atherosclerosis.2013.03.029.

[10] K. Tarasov, K. Ekroos, M. Suoniemi, D. Kauhanen, T. Sylvänne, R. Hurme, I. Gouni-Berthold, H.K. Berthold, M.E. Kleber, R. Laaksonen, W. März, Molecular lipids identify cardiovascular risk and are efficiently lowered by simvastatin and PCSK9 deficiency, J. Clin. Endocrinol. Metab. 99 (2014) E45-E52, https://doi.org/10.1210/jc.2013-2559.

[11] A. Baruch, S. Mosesova, J.D. Davis, N. Budha, A. Vilimovskij, R. Kahn, K. Peng, K.J. Cowan, L.P. Harris, T. Gelzleichter, J. Lehrer, J.C. Davis, W.G. Tingley, Effects of RG7652, a monoclonal antibody against PCSK9, on LDL-C, LDL-C subfractions, and inflammatory biomarkers in patients at high risk of or with established coronary heart disease (from the phase 2 EQUATOR study), Am. J. Cardiol. (2017), https://doi.org/10.1016/j.amjcard.2017.02.020.

[12] R.J. Havel, H.A. Eder, J.H. Bragdon, The distribution and chemical composition of ultracentrifugally separated lipoproteins in human serum, J. Clin. Invest. 34 (1955) 1345-1353, https://doi.org/10.1172/JCI103182.

[13] K. Ekroos, Unraveling Glycerophospholipidomes by Lipidomics, Humana
Press, Totowa, NJ, 2008, https://doi.org/10.1007/978-1-59745-463-6_17.

[14] J. Folch, M. Lees, G.H. Sloane Stanley, A simple method for the isolation and purification of total lipides from animal tissues, J. Biol. Chem. 226 (1957) 497-509.

[15] M. Ståhlman, C.S. Ejsing, K. Tarasov, J. Perman, J. Borén, K. Ekroos, Highthroughput shotgun lipidomics by quadrupole time-of-flight mass spectrometry, J. Chromatogr. B. Analyt. Technol. Biomed. Life Sci. 877 (2009) 2664-2672, https://doi.org/10.1016/j.jchromb.2009.02.037.

[16] K. Ekroos, I.V. Chernushevich, K. Simons, A. Shevchenko, Quantitative profiling of phospholipids by multiple precursor ion scanning on a hybrid quadrupole time-of-flight mass spectrometer, Anal. Chem. 74 (2002) 941-949.

[17] K. Ekroos, C.S. Ejsing, U. Bahr, M. Karas, K. Simons, A. Shevchenko, Charting molecular composition of phosphatidylcholines by fatty acid scanning and ion trap MS3 fragmentation, J. Lipid Res. 44 (2003) 2181-2192, https://doi.org/ 10.1194/jlr.D300020-JLR200.

[18] R. Laaksonen, K. Ekroos, M. Sysi-Aho, M. Hilvo, T. Vihervaara, D. Kauhanen, M. Suoniemi, R. Hurme, W. März, H. Scharnagl, T. Stojakovic, E. Vlachopoulou, M.L. Lokki, M.S. Nieminen, R. Klingenberg, C.M. Matter, T. Hornemann, P. Jüni, N. Rodondi, L. Räber, S. Windecker, B. Gencer, E.R. Pedersen, G.S. Tell, O. Nygård, F. Mach, J. Sinisalo, T.F. Lüscher, Plasma ceramides predict cardiovascular death in patients with stable coronary artery disease and acute coronary syndromes beyond LDL-cholesterol, Eur. Heart J. 37 (2016) 1967-1976, https://doi.org/10.1093/eurheartj/ehw148.

[19] P.J. Meikle, G. Wong, D. Tsorotes, C.K. Barlow, J.M. Weir, M.J. Christopher, G.L. MacIntosh, B. Goudey, L. Stern, A. Kowalczyk, I. Haviv, A.J. White, A.M. Dart, S.J. Duffy, G.L. Jennings, B.A. Kingwell, M. Weir, Plasma lipidomic analysis of stable and unstable coronary artery disease, Arterioscler. Thromb. Vasc. Biol. $31 \quad$ (2011) 2723-2732, https://doi.org/10.1161/ ATVBAHA.111.234096.

[20] A.S. Havulinna, M. Sysi-Aho, M. Hilvo, D. Kauhanen, R. Hurme, K. Ekroos, V. Salomaa, R. Laaksonen, Circulating ceramides predict cardiovascular outcomes in the population-based FINRISK 2002 cohort, Arterioscler. Thromb. Vasc. Biol. 36 (2016) 2424-2430, https://doi.org/10.1161/ ATVBAHA.116.307497.

[21] M.J. Koren, D. Kereiakes, R. Pourfarzib, D. Winegar, P. Banerjee, S. Hamon, C. Hanotin, J.M. McKenney, Effect of PCSK9 inhibition by alirocumab on lipoprotein particle concentrations determined by nuclear magnetic resonance spectroscopy, J. Am. Heart Assoc. 4 (2015), https://doi.org/10.1161/ sAHA.115.002224.

[22] A. Sahebkar, P. Di Giosia, C.A. Stamerra, D. Grassi, C. Pedone, G. Ferretti, T. Bacchetti, C. Ferri, P. Giorgini, Effect of monoclonal antibodies to PCSK9 on high-sensitivity C-reactive protein levels: a meta-analysis of 16 randomized controlled treatment arms, Br. J. Clin. Pharmacol. 81 (2016) 1175-1190, https://doi.org/10.1111/bcp.12905.

[23] E.A. Dennis, P.C. Norris, Eicosanoid storm in infection and inflammation, Nat Rev. Immunol. 15 (2015) 511-523, https://doi.org/10.1038/nri3859.

[24] T.W.K. Ng, E.M.M. Ooi, G.F. Watts, D.C. Chan, J.M. Weir, P.J. Meikle, P.H.R. Barrett, Dose-dependent effects of rosuvastatin on the plasma sphingolipidome and phospholipidome in the metabolic syndrome, J. Clin. Endocrinol. Metab. 99 (2014) E2335-E2340, https://doi.org/10.1210/jc.2014-1665. 